\title{
ON THE COVERING OF ANALYTIC LOCI BY COMPLEXES*
}

\author{
BY \\ B. O. KOOPMAN AND A. B. BROWN
}

1. Introduction. Classical analysis is frequently occupied with the varieties defined by analytic equations. Modern analysis situs, on the other hand, deals with complexes formed by the union of cells, and investigates the topology of these figures by combinatorial methods. In order that the results of this method be applicable to the analytic varieties, it is essential that a theorem be established, both in the real and in the complex domain, which states that analytic varieties may be obtained as complexes of cells. The proof of this theorem is the object of the present paper.

While the fact that analytic varieties belong to the complexes of analysis situs has been quite generally assumed on intuitive grounds, it has not up to now been given a rigorous general proof. In the case of algebraic varieties, van der Waerden $\dagger$ has given a proof which, by the nature of the case, cannot be extended to analytic varieties in general. An outline of a proof has been given in the general case by Lefschetz. $\ddagger$ But examination reveals that this discussion is incomplete. Thus (to mention only two of the logical diffculties) the statement, page 365 , that "the conditions for coincidence ... are expressed by the vanishing of certain functions holomorphic . . ." is true only in the small. These functions, determined at two different points, are not in general analytically continuable into each other. Again, the use of projections requires the existence of a unique direction for application of the Weierstrass preparation theorem for every point of a locus, a result difficult to demonstrate (cf. our Theorem 5.I).

At the end of the paper we establish a theorem for the space of a set of $n$ complex variables, and finally show that in the real case the $(n-1)$-dimensional part of any locus defined by the vanishing of analytic functions is either vacuous or an orientable $(n-1)$-cycle $(\bmod 2)$.

2. Some properties of real analytic functions. The theorems of this section are for the most part corollaries to theorems about complex quantities.

* Presented to the Society, October 31, 1931, under the title Covering of analytical loci by complexes; received by the editors November 16, 1931.

$\dagger$ B. L. van der Waerden, Topologische Begründung des Kalküls der abzählenden Geometrie, Anhang 1, Mathematische Annalen, vol. 102 (1929), p. 360.

‡ S. Lefschetz, Topology, pp. 362-366. Colloquium Series, vol. 12; New York, 1930. 
Unless otherwise stated, notations are as in Osgood's Funktionentheorie, vol. $\mathrm{II} \dagger$ (hereafter referred to as Osgood II). When we restrict ourselves to real functions throughout, we denote properties by adding " $R$," as "equivalent$R$," "reducible- $R$ ", denoting the ordinary properties, in the domain of complex numbers, by "equivalent- $C$ ", etc. The conjugate of an analytic function is the function which, for real values of the variables, takes on values conjugate complex to those of the given function, and is denoted by placing a dash over the symbol for the function.

We observe that any function of $x_{1}, \cdots, x_{n}$ which is analytic for real $(x)$ can be put in the form $A+i B$, where $A$ and $B$ are real and analytic. The real locus where the function vanishes is the real locus where $A=B=0$, or where $A^{2}+B^{2}=0$. Consequently, the problem of investigating the nature of the real locus where any set of analytic functions vanishes is equivalent to that in which the functions are all real. We consider the latter case henceforth.

In the theorems of this section, the independent variables are understood to be $x_{1}, \cdots, x_{n}$. We omit proof of the following lemma.

Leмma 2.I. If $A=B C$ in some real neighborhood, $A, B, C$ are analytic, $A$ and $B$ are real, and $A$ is not identically zero, then $C$ is real.

CoROLLARY 2.II. If two real functions are equivalent- $C$ at a point $P$, they are equivalent- $R$ at $P$.

If both functions are identically zero, the result is obvious. If not, it is a consequence of Lemma 2.I. (Equivalence is defined in Osgood II, Chapter 2, \$4.)

LEMMA 2.III. If $F$ is analytic at a real point $P$, and not equivalent there to any real analytic function, then $F$ is not equivalent to $\bar{F}$.

From $F=\Omega \bar{F}$ would follow $\bar{F}=\bar{\Omega} F$, hence $F=\Omega \bar{\Omega} F$. Since $F$ cannot be identically zero, $\Omega \bar{\Omega}=1$, so that $\Omega=e^{i k}, k$ a real constant. On writing $F^{*}=e^{-i k / 2} F$, we have $F$ equivalent to $F^{*}$, which is real since

$$
\bar{F}^{*}=e^{i k / 2} \bar{F}=e^{i k / 2} e^{-i k} F=e^{-i k / 2} F=F^{*} .
$$

As this contradicts the hypotheses, $F$ cannot be equivalent to $\bar{F}$.

THEOREM 2.IV. If $A$ is real, analytic, zero at the real point $P$, irreducible- $R$ there but reducible- $C$, and $B$ is an irreducible- $C$ factor of $A$ at $P$, then $\bar{B}$ is irreducible- $C$ at $P, B$ and $\bar{B}$ are not equivalent at $P$, and

$$
A=B \bar{B} \Omega
$$

near $P$, where $\Omega$ is real, analytic, and not zero at $P$.

† W. F. Osgood, Lehrbuch der Funktionentheorie, vol. II, second edition, Leipzig, 1929. 
From $\bar{B}=B_{1} B_{2}$, with $B_{1}$ and $B_{2}$ analytic and zero at $P$, would follow $B=\bar{B}_{1} \bar{B}_{2}$, with $\bar{B}_{1}$ and $\bar{B}_{2}$ analytic and zero at $P$, contrary to the hypothesis that $B$ is irreducible- $C$ at $P$. Hence the first conclusion is valid.

By hypothesis, we have $A=B D$, where $D$ is analytic and zero at $P$.

Since $A$ is irreducible- $R, B$ is not equivalent to any real function. From Lemma 2.III we then infer that $B$ is not equivalent to $\bar{B}$.

Since $A$ is real, $A=\bar{A}=\bar{B} \bar{D}$, so that $\bar{B}$ divides $A$ at $P$. (Since $P$ is a real point, $\bar{B}$ is zero at $P$.) Since $B$ is irreducible- $C$ and is not equivalent- $C$ to $\bar{B}$, so that $B$ and $\bar{B}$ have no common divisor, it follows that $\dagger$

$$
A=B \bar{B} \Omega \text {, }
$$

where $\Omega$ is analytic at $P$. Since $B \bar{B}$ is real, it follows from Lemma 2 .I that $\Omega$ is real. If $\Omega$ were zero at $P$, then by (2.1) $A$ would be reducible- $R$ at $P$, contrary to hypothesis. Hence $\Omega \neq 0$ at $P$. This completes the proof.

Theorem 2.V. If $A$ is real, analytic, zero at $P$ but not identically zero, it can be factored in one and only one way into a product of real analytic factors zero at $P$ and irreducible- $R$ there, if we do not distinguish between equivalent factors. Those of the factors that are not equivalent to real analytic functions can be paired so that each member of a pair is equivalent-C to the conjugate of the other member of the pair.

By use of the theorem of unique factorability in the domain of complex quantities, $\ddagger$ and our Theorem 2.IV and Lemma 2.I, the proof is easily constructed. We shall give no further details.

COROLLARY 2.VI. Factorization of a real singular algebroid polynomial into irreducible- $R$ factors which are real singular algebroid polynomials with the same vertex, is unique, provided we do not distinguish between equivalent factors. The factors which are not equivalent to real functions can be paired as in Theorem 2.V.

The result follows easily from Theorem $2 . \mathrm{V}$ and $\$ 7$ (Satz 2) and $\$ 3$ in Osgood II, Chapter 2.

We shall use the usual definitions of resultant and discriminant, say as defined in Perron's Algebra. $\$$ The definitions in Bôcher's\| Algebra differ from these merely by constant multiples depending only on the degrees of the polynomials involved. Since the resultant and discriminant to be used vanish at the same points as the resultant and discriminant defined in Osgood II

\footnotetext{
† Osgood II, Chapter 2, $\$ 4$, Hauptsatz.

$\ddagger$ Osgood II, Chapter 2, \$5, Hauptsatz.

$\S$ O. Perron, Algebra, vol. I, pp. 218, 225; Berlin, de Gruyter, 1927.

|| M. Bôcher, Introduction to Higher Algebra, pp. 195, 250; New York, Macmillan, 1907.
} 
(Chapter 2, §9), the theorems in Osgood II regarding their vanishing remain valid under the present notations.

Theorem 2.VII. Let $F$ be a real, singular algebroid polynomial (ausgezeichnet Pseudopolynom). If its discriminant vanishes identically, then $F$ is a product of real singular algebroid polynomials of lower degrees, with the same vertex, one of which occurs as a multiple factor.

This theorem is proved easily by use of the corresponding theorem for the complex case, ${ }^{*}$ and our Lemma 2.I and Corollary 2.VI. Theorem 2.VIII, now following, is proved in similar manner, and we give no further proof for either theorem.

THEOREM 2.VIII. If $F$ and $G$ are real singular algebroid polynomials with the same vertex, the identical vanishing of their resultant implies that they have a common real singular algebroid factor.

COROLLARY 2.IX. Theorems 2.VII and 2.VIII are also valid for non-singular algebroid polynomials, providing the coefficient of the highest power is not zero at the vertex.

By the method of Chapter 2, $\$ 10$, in Osgood II, it is easily shown that such an algebroid polynomial is a product of one or more algebroid polynomials each of which has all its roots at the vertex coincident. Consider, say, Theorem 2.VII. The discriminant of the product equals the product of the discriminants of the factors by the squares of the resultants of the pairs of factors. $\dagger$ At least one of these discriminants and resultants must vanish identically; and if it is a resultant, the two corresponding factors must have equal roots at the vertex. The proof is now easily completed by use of Theorem 2.VII or 2.VIII, and the facts that an algebroid polynomial with roots all coincident at its vertex is expressible as a singular algebroid polynomial in a new variable equal to a constant plus the original one; and that, as follows from the expressions for discriminants and resultants in terms of differences of roots, $\ddagger$ this new polynomial has the same discriminant (or two of them have the same resultant).

LEMma 2.X. Let $F\left(x_{1}, \cdots, x_{m} ; y ; z\right)=F(x, y, z)$ be an algebroid polynomial with vertex at the origin:

$$
F(x, y, z)=z^{N}+\psi_{1}(x, y) z^{N-1}+\cdots+\psi_{N}(x, y)
$$

* Osgood II, Chapter 2, $\S$, Satz 3. It is also convenient to use $\S 3$, and $\S 5$, Satz 3 and Zusatz. In the proof of Theorem 2.VIII we would use $\$ 9$, Satz 1.

† Perron, loc. cit., p. 227, formula (10). For Theorem 2.VIII we would use formula (21), p. 223, giving the resultant as the product of the resultants of the factors.

$\ddagger$ Perron, loc. cit., p. 275, formula (8), and p. 278, formula (15). 
suppose that it is irreducible-C at the origin, and that its coefficients are analytic in the $(2 m+2)$-dimensional neighborhood $E:\left|x_{i}\right|<e,|y|<e$; finally, let the discriminant $R(x, y)$ of $F(x, y, z)$ vanish only on the locus $y=0$. Then we shall have, for all $\left|x_{i}\right|<e$,

$$
F(x, 0, z)=[z-\Upsilon(x)]^{N},
$$

where $\Upsilon$ is analytic for all $\left|x_{i}\right|<e$.

First we observe that at any point of $E$ not on the locus $y=0$, any root can be continued analytically into any other at the point, along a curve in $E$ not meeting the locus $y=0$. This is proved as in Osgood II, Chapter $2, \S 10$.

Suppose now that at $P$ on the locus $y=0, F$ had two distinct roots. It follows from the continuity of algebroid functions that two roots could then be found at a point $Q$ near $P$, not on $y=0$, which could not be continued into each other near $P$. But from the preceding paragraph it follows that a path could be described from $Q$ to the neighborhood of the origin and back to $Q$, not meeting the locus $y=0$, and such that along it one of the roots in question is continued analytically into the other. This path could be deformed into a curve near $P$, so as still to pass through $Q$, without meeting the locus $y=0$ during the deformation. The result would be a curve from $Q$ to $Q$, near $P$, along which the one root could be continued into the other. As this would contradict the statement above in this paragraph, it follows that at every point $(x, 0)$ in $E$, all the roots of $F$ coincide.

We then infer that $F(x, 0, z)$ has the form $(2.3)$, for any point $(x, 0)$ in $E$. Comparing with (2.2), we find that $\Upsilon(x)=-\psi_{1}(x, 0) / N$, hence is analytic. This completes the proof.

3. Lemmas of analysis situs. We present the following lemmas:

LEMMA 3.I. Given a complex and a number of sub-complexes, then the sum of the sub-complexes is a sub-complex, and the intersection of the sub-complexes is a sub-complex.

LEMMA 3.II. Let $E_{n}$ and $S_{n-1}$ be an $n$-cell and its boundary, in the space of the variables $\left(x_{1}, \cdots, x_{n}\right)$, homeomorphic to an ordinary $(n-1)$-sphere and its interior in a euclidean $n$-space. Let $y_{1}$ and $y_{2}$ be two functions of $(x)$ single-valued and continuous over $\left(E_{n}+S_{n-1}\right)$, such that $y_{2}>y_{1}$ on $E_{n}$, and $y_{2} \geqq y_{1}$ on $S_{n-1}$. Then the locus, say $H$, of points in $(x, y)$-space for which $(x)$ is on $E_{n}$ or $S_{n-1}$ and $y_{1} \leqq y \leqq y_{2}$, is homeomorphic to an ordinary $n$-sphere and its interior in euclidean $(n+1)$-space, in such a way that the interior of the $n$-sphere corresponds to the points for which $(x)$ is on $E_{n}$ and $y_{1}<y<y_{2}$.

We omit the proof of Lemma 3.I, and proceed to prove Lemma 3.II. 
Since $H$ may be replaced by a homeomorph, we may assume first that $S_{n-1}$ and $E_{n}$ are an ordinary $(n-1)$-sphere and its interior, in the $(x)$-plane, say with center at the origin. Next, we redefine $y_{1}$ and $y_{2}$ as follows. They are unchanged over $S_{n-1}$. At the origin, $y_{2}$ takes on a positive value greater than at any point on $S_{n-1}$, determining a point, say $P$, on the $y$-axis; and $y_{1}$ takes on a negative value less than at any point of $S_{n-1}$, determining a point $Q$ on the $y$-axis. For other values of $(x), y_{2}$ is determined by the surface obtained by joining $P$ by straight line segments to all the points $\left(x, y_{2}\right)$ for which $(x)$ is on $S_{n-1}$; and $y_{1}$ is similarly defined, with the point $Q$ replacing $P$.

The new $H$ is homeomorphic to the old, with interiors corresponding, and furthermore it is convex from the origin. For, if we consider any straight line from the origin (not the $y$-axis), it and the $y$-axis determine a 2-plane, which cuts out from $H$ a convex 2-dimensional region, bounded by four, five or six straight sides, four of which are not parallel to the $y$-axis. The line segment cuts the boundary of this figure in just one point; hence also cuts the boundary of $H$ in just one point. These line segments (including the two along the $y$-axis) set the boundary of $H$ in a one-to-one continuous correspondence with any $n$-sphere with center at the point $(0,0)$; which is extended in an obvious manner to the interiors. (Cf. Lefschetz, loc. cit., p. 9.) This completes the proof.

4. Nature of an analytic locus in the small. By an analytic locus we mean one defined by equating to zero certain functions analytic in the space-coördinates. As a means of finding the nature of such a locus, we introduce an algorithm.

We begin with a finite set of functions

$$
\Theta_{1}\left(x_{1}, \cdots, x_{n}\right), \cdots, \Theta_{s}\left(x_{1}, \cdots, x_{n}\right),
$$

which are (i) analytic at the origin $(x)=(0)$; (ii) real; (iii) not identically zero.

The first step of the algorithm is a rotation of axes such that, for the new variables (using the same notations for the variables and functions), we have

$$
\Theta_{i}\left(0, \cdots, 0, x_{n}\right) \not \equiv 0 \quad(i=1,2, \cdots, s) .
$$

Next we apply a theorem of Weierstrass, ${ }^{*}$ giving us, near (0),

$$
\Theta_{i}\left(x_{1}, \cdots, x_{n}\right) \equiv W_{i}^{(n)}\left(x_{1}, \cdots, x_{n}\right) \cdot \prod_{k}\left[F_{k}^{(n)}\left(x_{1}, \cdots, x_{n}\right)\right]^{P_{k}} .
$$

Here $W_{i}^{(n)}$ is real, analytic and not zero at the origin, the product is finite, and $F_{k}^{(n)}$ is an irreducible- $R$ singular algebroid polynomial with vertex at $(0)$; that is, it has the general form

\footnotetext{
* Osgood II, Chapter 2, \$2.
} 


$$
F_{k}^{(n)} \equiv x_{n}^{N}+\psi_{1}\left(x_{1}, \cdots, x_{n-1}\right) x_{n}^{N-1}+\cdots+\psi_{N}\left(x_{1}, \cdots, x_{n-1}\right),
$$

where the $\psi$ 's are real, analytic and zero at $(x)=(0)$; but $F_{k}^{(n)}$ is not factorable into a product of two such functions. The notation in (4.2) and (4.3) is generic and not intended to be complete; thus, $N$, $\psi_{1}$, etc., will usually be different for different values of $k$. The superscripts are used here, as throughout, to indicate the number of independent variables. The exponents $P_{k}$ and $N$ are positive integers, but if $\Theta_{i}(0) \neq 0$, the product $\Pi_{k}$ is replaced by unity. Finally, (4.2), which holds for $i=1,2, \cdots, s$, defines a finite set of real $F^{(n)}$ 's, which we have arranged in an arbitrary order as

$$
F_{1}^{(n)}, F_{2}^{(n)}, \cdots, F_{t}^{(n)},
$$

where no two of these $F$ 's are equivalent at (0). (See Corollary 2.VI.)

Next we form the discriminants $R_{i i^{(n-1)}}\left(x_{1}, \cdots, x_{n-1}\right)$ of all $F_{i}{ }^{(n)}$ 's in (4.4), and the resultants $R_{i j}^{(n-1)}\left(x_{1}, \cdots, x_{n-1}\right)$ of all the pairs $F_{i}^{(n)}, F_{i}^{(n)}$, in (4.4). Let these discriminants and resultants be denoted by

$$
\Theta_{1}^{(n-1)}\left(x_{1}, \cdots, x_{n-1}\right), \quad \Theta_{2}^{(n-1)}\left(x_{1}, \cdots, x_{n-1}\right), \cdots,
$$

a finite set. None of them vanishes identically, as follows from Theorems 2.VII and 2.VIII.

This completes the first step in our algorithm, which consisted in (1) choosing axes so that (4.1) is satisfied; (2) determining the functions (4.4); (3) determining the functions (4.5). We now proceed to apply the same process to the functions $\Theta_{k}^{(n-1)}\left(x_{1}, \cdots, x_{n-1}\right)$, to determine a new set of functions $\Theta_{k}^{(n-2)}\left(x_{1}, \cdots, x_{n-2}\right)$. We note that the change of axes coming at the beginning of this process will involve only the variables $x_{1}, \cdots, x_{n-1}$, hence will not affect what was done in the first step of the algorithm.

We then repeat the process, doing it $(n-1)$ times in all. The general equations replacing (4.2) and (4.3) are

$$
\begin{aligned}
\left., x_{v}\right) & \equiv \Theta_{l}^{(\nu)}\left(x_{1}, \cdots, x_{v}\right) \\
& \equiv W_{l}^{(\nu)}\left(x_{1}, \cdots, x_{v}\right) \prod_{k}\left[F_{k}^{(\nu)}\left(x_{1}, \cdots, x_{v}\right)\right]^{P_{k}} \\
F_{i}^{(\nu)} & \equiv x_{\nu}^{N}+\psi_{1}\left(x_{1}, \cdots, x_{\nu-1}\right) x_{\nu}^{N-1}+\cdots+\psi_{N}\left(x_{1}, \cdots, x_{\nu-1}\right) \\
& (\nu=1, \cdots, n) .
\end{aligned}
$$

At the termination of this process, we complete our algorithm by determining $n$ positive constants $\mathfrak{a}_{1}, \cdots, \mathfrak{a}_{n}$, and, corresponding with these, $n$ real closed neighborhoods $\mathfrak{A}^{\nu}(\nu=1, \cdots, n)$ :

$\mathfrak{A}^{\nu}$ :

$$
\left|x_{k}\right| \leqq \mathfrak{a}_{k}
$$$$
(k=1, \cdots, \nu),
$$ 
having the following properties: $(\alpha)$ The functions $R^{(\nu)}$ or $\Theta^{(\nu)}, W^{(\nu)}$, and $F^{(v)}$ are analytic in $\mathfrak{A}^{\nu} .(\beta)$ Throughout $\mathfrak{A}^{\nu}, W^{(\nu)} \neq 0 .(\gamma)$ If, for $\nu<n$, $\left(x_{1}, \cdots, x_{\nu}\right)$ is in $\mathfrak{A}^{\nu}$, each root $x_{\nu+1}$ of $F_{i}^{(\nu+1)}=0$ is restricted by the inequality $\left|x_{v+1}\right|<\mathfrak{a}_{v+1}$. The $\mathfrak{a}^{\prime}$ 's are determined as follows. First, take $\mathfrak{a}_{n}$ such that properties $(\alpha),(\beta)$ for $\mathfrak{A}^{n}$ hold in the neighborhood $\mathfrak{A}^{\prime n}:\left|x_{1}\right| \leqq \mathfrak{a}_{n}, \cdots$, $\left|x_{n}\right| \leqq \mathfrak{a}_{n}$; second take $\mathfrak{a}_{n-1}<\mathfrak{a}_{n}$ and such that $(\alpha),(\beta),(\gamma)$ for $\mathfrak{A}^{n-1}$ hold in the neighborhood $\mathfrak{U}^{\prime n-1}:\left|x_{1}\right| \leqq \mathfrak{a}_{n-1}, \cdots,\left|x_{n-1}\right| \leqq \mathfrak{a}_{n-1}$; property $(\gamma)$ being obtainable by virtue of the continuity of algebroid functions. By repeating this process $n$ times, the desired set of constants is obtained.

The following theorem, which is fundamental for the later results, is valid for the values $\nu=1,2, \cdots, n-1$.

THEOREM 4.I ${ }^{\nu}$. (1) The closed region $\mathfrak{A}^{\nu}$ can be covered by a complex $A^{\nu}$ of analytic cells $a^{\mu}(\mu=0,1, \cdots, \nu)$.

(2) Each cell $a^{\mu}, 0<\mu<\nu$, is mapped on a $\mu$-cell $b^{\mu}$ in the $\mu$-space of a certain set of $\mu x^{\prime} s, x_{t_{1}}, \cdots, x_{t_{\mu}}$, by equations

$$
\begin{gathered}
x_{t_{\mu+1}}=\omega_{1}\left(x_{t_{1}}, \cdots, x_{t_{\mu}}\right), \\
x_{t_{\nu}}=\omega_{\nu-\mu}\left(x_{t_{1}}, \cdots, x_{t_{\mu}}\right),
\end{gathered}
$$

where the functions $\omega$, which are determined by $a^{\mu}$, are real and analytic in $b^{\mu}$, and continuous on $b^{\mu}$ and its boundary. Each cell $a^{\nu}$ is a region of $\mathfrak{A}^{\nu}$.

(3) If $\Phi_{i v}{ }^{\left({ }^{+1}+1\right)}\left(x_{t_{1}}, \cdots, x_{t_{\mu}} ; x_{v+1}\right)$ is the polynomial obtained from $F_{i}{ }^{\left({ }^{\nu+1}\right)}$ by the substitution $\left(4.8^{\nu}\right)$ (or if $\nu=\mu, \Phi_{i \nu}{ }^{(\mu+1)}=F_{i}^{(\nu+1)}$ ), the following reduction will take place throughout $b^{\mu}$ :

$$
\Phi_{i \nu}^{(\mu+1)}=\prod_{\rho}\left[x_{\nu+1}-\Omega_{i \rho}\left(x_{t_{1}}, \cdots, x_{t_{\mu}}\right)^{N_{i \rho}}\right],
$$

where the $\Omega^{\prime}$ s are analytic within $b^{\mu}$ (but not necessarily real).

(4) If $\Omega_{i \rho}$ and $\Omega_{j \sigma}$ are equal at one point of $b^{\mu}$, they will be equal identically.

The proof is by induction. Let us assume Theorem 4.I ${ }^{\nu}$, with $\nu \leqq n-2$, and proceed to prove Theorem 4.I ${ }^{\nu+1}$. As the proof that we are to give will also hold for the value $\nu=1$ with no assumptions at all (but with certain simplifications, which we shall not mention), the proof will establish the theorem.

In the first place, the functions $\Omega_{i \rho}$ belonging to $a^{\mu}$, together with a set of functions $\Omega_{i \sigma}$ one belonging to each of the cells on the boundary of $b^{\mu}$, define, in all, a function continuous over $b^{\mu}$ and its boundary. (If $\mu=\nu$, we use $b^{\nu}$ as another name for $a^{\nu}$.) To prove this, we observe that all the functions $\Omega_{i p}$ and $\Omega_{i \sigma}$, being algebroid, form a set continuous over $b^{\mu}$ and its boundary, hence uniformly continuous. It follows that if we extend the function $\Omega_{i \rho}$ so as to 
assume, over the boundary of $b^{\mu}$, its limiting values, the resulting function will be continuous over $b^{\mu}$ and its boundary. That the values so defined for a single cell of the boundary are those of one of the $\Omega_{i \sigma}$ 's for that cell follows from the facts that the values defined are continuous over the cell in question; the value at any given point of the cell must be that of one of the $\Omega_{i \sigma}$ 's; and the functions $\Omega_{i \sigma}$ satisfy condition (4) of the theorem. Hence the italicized statement is correct.

Next, we note that if $\Omega_{i \rho}$ is real at one point of $b^{\mu}$, it is real at every point of $b^{\mu}$. To prove this, we note that from condition (4) of Theorem 4.I ${ }^{\nu}$, the fact that the non-real roots of $\Phi_{i}{ }^{(\mu+1)}=0$ occur in conjugate imaginary pairs, and the continuity of the functions $\Omega_{i \rho}$, it follows that the functions $\Omega_{i \rho}$ that are not real occur in conjugate imaginary pairs. Since at no point, by condition (4) of Theorem 4.I ${ }^{\nu}$, can one of the latter functions coincide with its conjugate, it cannot take on any real values. On the other hand, the real functions take on only real values. Thus the statement is proved.

By an analytic cell we mean either a cell which is a region of the space of a certain set of variables, or one defined by equations like $\left(4.8^{\nu}\right)$.

We now proceed to divide the region $\mathfrak{A}^{\nu+1}$ into analytic cells. Consider the points $\left(x_{1}, \cdots, x_{v+1}\right)$ of $\mathfrak{A}^{\nu+1}$ whose projections $\left(x_{1}, \cdots, x_{v}\right)$ in $\mathfrak{A}^{\nu}$ lie on the cell $a^{\mu}$. We distribute these points into three classes of cells, as follows: First, those points for which $x_{v+1}=\Omega_{i \rho}\left(x_{t_{1}}, \cdots, x_{t_{\mu}}\right)$ will lie, for each real $\Omega$ of $a^{\mu}$, on certain analytic cells. Second, those points for which $x_{v+1}=\mathfrak{a}_{v+1}$ and those for which $x_{v+1}=-\mathfrak{a}_{v+1}$ will lie, respectively, on two analytic cells. No two of the cells thus far described can have a point in common, as follows from Theorem $4 . I^{\nu},(4)$, and the fact that $F_{i}^{(\nu+1)} \neq 0$ when $x_{v+1}= \pm a_{v+1}$. Finally, the cells of the third class consist of the points between successive pairs of cells of the first two classes, that is, points for which, respectively,

$$
\begin{aligned}
& -\mathfrak{a}_{v+1}<x_{v+1}<\Omega_{1}, \\
& \Omega_{1}<x_{p+1}<\Omega_{2}, \\
& \Omega_{k}<x_{v+1}<\mathfrak{a}_{v+1} .
\end{aligned}
$$

Here $\Omega_{1}, \cdots, \Omega_{k}$ denote the totality of real distinct $\Omega^{\prime}$ s of $a^{\mu}$, taken in such an order that at one point, and hence, by Theorem $4 . I^{\nu}$, (4), at every point, of $b^{\mu}$ we have

$$
\Omega_{1}<\Omega_{2}<\cdots<\Omega_{k} .
$$

By applying this construction for every cell of $A^{\nu}$, we decompose $\mathfrak{A}^{v+1}$ into a set $A^{n+1}$ of analytic cells, no two of which have a point in common. Each cell of either of the first two classes and its boundary, is in one-to-one con- 
tinuous correspondence with a cell and its boundary, of $A^{\nu}$, as follows from the construction and the italicized statement at the beginning of the proof of the theorem. From Theorem 4.I ${ }^{\nu},(4)$, and this same statement it follows that the hypotheses of Lemma 3.II are satisfied for each cell of the third class and its boundary; consequently each such boundary and $k$-cell are homeomorphic to an ordinary $(k-1)$-sphere and interior in a euclidean $k$-space. It follows that the set of cells $A^{v+1}$ forms a complex which, by a single regular subdivision, can be made simplicial. Thus (1) and (2) of Theorem 4.I ${ }^{v+1}$ are proved.

We shall now prove (3) and (4) of Theorem 4.I ${ }^{\nu}$ for the neighborhood of a point, after which both are easily obtained for the entire cell $b^{\mu}$.

Let $\Phi_{i}{ }^{*(\mu+2)}$ and $P_{i j}{ }^{(\mu+1)}$ be the algebroid polynomials obtained from $F_{i}^{\left({ }^{(\nu+2)}\right.}$ and $R_{i j}{ }^{\left({ }^{(v+1)}\right.}$, respectively, as a result of making the substitution $\left(4.8^{\nu}\right)$. (If $\mu=\nu$, this amounts simply to renaming the functions.) Then $P_{i j}{ }^{(\mu+1)}$ will

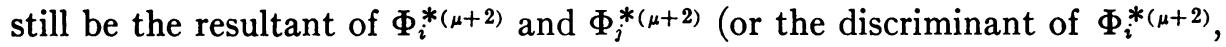
if $i=j$ ). Furthermore, by (4.6) and Theorem 4.I ${ }^{p}$, (3), we have

$$
P_{i j}^{(\mu+1)}=U \prod_{k} \prod_{r}\left[x_{\nu+1}-\Omega_{k r}\left(x_{t_{1}}, \cdots, x_{t_{\mu}}\right)\right]^{M_{k r}}
$$

( $U$ analytic and not zero in $b^{\mu}$ ). Now let $a$ be any cell of $A^{\nu+1}$ projecting on $A^{\nu}$ in $b^{\mu}$, and take any point $\left(x^{0}\right)=\left(x_{1}^{0}, \cdots, x_{\nu+1}^{0}\right)$ in $a$. Suppose that at $\left(x^{0}\right)$ the following reduction to irreducible- $C$, though not necessarily distinct, factors $\phi$ takes place:

$$
\Phi_{i}^{*(\mu+2)}=\prod_{\alpha} \phi_{i \alpha}^{(\mu+2)},
$$

where $\phi$ is an algebroid polynomial in $x_{v+2}$ with vertex at $\left(x^{0}\right)$, in general not singular. Then its roots at $\left(x^{0}\right)$ are all equal.

In (4.10), if the discriminants and resultants of the $\phi$ 's are denoted by $\rho_{i \alpha, i \alpha}$ and $\rho_{i \alpha, j \beta}$ as usual, we shall have

$$
P_{i j}^{(\mu+1)}= \pm \prod_{\alpha} \prod_{\beta} \rho_{i \alpha, j \beta}^{(\mu+1)} \quad(i=j \text { or } i \neq j),
$$

in a neighborhood of $\left(x^{0}\right)$. This follows from the formulas for the discriminant and resultant of the product of a number of polynomials. $\dagger$ An expression $x_{v+1}-\Omega_{k r}\left(x_{t_{1}}, \cdots, x_{t_{\mu}}\right)$ in (4.9) vanishes at $\left(x^{0}\right)$ if and only if (1) $a$ is a cell of the first class (cf. above construction); (2) $\Omega_{k r}$ is the $\Omega_{\rho}$ used in its construction. It then follows by (4.9) and (4.11) and the theorem of unique factorability that if $\rho_{i \alpha, j \beta}^{(\mu+1)}=0$ at $\left(x^{0}\right)$, it has there the form

† Perron, loc. cit., p. 223, formula (21), and p. 227, formula (10).

$\ddagger$ Osgood II, Chapter 2, §7, Satz 1. 


$$
\rho_{i \alpha, \beta \beta}^{(\mu+1)}=V\left[x_{\nu+1}-\Omega_{\rho}\right]^{K},
$$

where $V$ is analytic and not zero at $\left(x^{0}\right)$.

The proof of Theorem 4.I ${ }^{\nu+1}$, (3), for the neighborhood of $\left(x^{0}\right)$, now follows. If $a$ is of the second class, $\left(4.8^{v+1}\right)$ is defined as $\left(4.8^{\nu}\right)$ together with the equation $x_{v+1}=\mathfrak{a}_{v+1}$ or $x_{v+1}=-\mathfrak{a}_{v+1}$; if $a$ is of the third class, $\left(4.8^{v+1}\right)$ is the same as $\left(4.8^{\nu}\right)$, but with $x_{v+1}$ considered as one of the independent variables; if $a$ is of the first class, $\left(4.8^{v+1}\right)$ is taken as $\left(4.8^{v}\right)$ together with the additional equation

$$
x_{\nu+1}=\Omega_{\rho}\left(x_{t_{1}}, \cdots, x_{t_{\mu}}\right)=\omega_{\nu-\mu+1}\left(x_{t_{1}}, \cdots, x_{t_{\mu}}\right) .
$$

In all cases the function $\phi_{i \alpha}{ }^{(\mu+2)}$ becomes, after the above substitution $\left(4.8^{v+1}\right)$, an algebroid polynomial in $x_{v+2}$, with coefficients depending on $\left(x_{t_{1}}, \cdots, x_{t_{\mu}}\right)$ or on $\left(x_{t_{1}}, \cdots, x_{t_{\mu}}, x_{\nu+1}\right)$. We say that it reduces at $\left(x^{0}\right)$ to the form

$$
\phi_{i \alpha}^{(\mu+\sigma)}=\left(x_{\nu+2}-\Upsilon\right)^{M} \quad(\sigma=1 \text { or } 2),
$$

where $\Upsilon$ is analytic at $\left(x^{0}\right)$ in $\left(x_{t_{1}}, \cdots, x_{t_{\mu}}\right)$ or in $\left(x_{t_{1}}, \cdots, x_{t_{\mu}}, x_{v+1}\right)$. This is clearly true if $\rho_{i \alpha, i \alpha}^{(\mu+1)}\left(x^{0}\right) \neq 0$, as in that case, since $\partial \phi_{i \alpha} / \partial x_{v+2}$ cannot be zero where $\phi_{i \alpha}=0$, (4.13) follows by the implicit function theorem, with $M=1 . \dagger$ If $\rho_{i \alpha, i \alpha}^{(\mu+1)}\left(x^{0}\right)=0$, then (4.12) will be valid, and if we introduce the new variable $y=x_{v+1}-\Omega_{\rho}$ in place of $x_{v+1}$, the result follows from Lemma 2.X. As every function $\Phi_{i, v+1}$ of Theorem 4.I ${ }^{\nu+1},(3)$, is a function $\Phi_{i}^{*}$, from (4.13) and (4.10) it follows that condition (3) of Theorem $4 . I^{\nu+1}$ is satisfied for a neighborhood of $\left(x^{0}\right)$, taking the functions $\Omega_{i \rho}$ as the functions $\Upsilon$.

To prove Theorem 4.I ${ }^{v+1},(4)$, for a neighborhood of $\left(x^{0}\right)$, suppose that $\Upsilon$ and $\Upsilon^{\prime}$, corresponding to $\phi_{i \alpha}{ }^{(\mu+1)}$ and $\phi_{j \beta}{ }^{(\mu+1)}$ respectively, as in (4.13), are equal at $\left(x^{0}\right)$. It follows that the resultant $\rho_{i \alpha, j \beta}\left(x^{0}\right)=0$. From (4.9) and (4.11) we infer that at the point $\left(x^{0}\right), x_{v+1}$ must equal one of the $\Omega_{k r}$ 's mentioned in (4.9). Hence $\left(x^{0}\right)$ must be on a cell of the first class; and consequently $\rho_{i \alpha, j \beta}$ has the form (4.12) near $\left(x^{0}\right)$. Since, as stated before (4.12), the $\Omega_{\rho}$ appearing in (4.12) is the one used in the construction of the cell $a$, it follows from (4.12) that the resultant in question vanishes at every point of a real neighborhood of $\left(x^{0}\right)$ on the cell. Hence $\phi_{i \alpha}{ }^{(\mu+1)}$ and $\phi_{j \beta^{(\mu+1)}}$ have a common root at every point of the neighborhood, and therefore, by (4.13), $\Upsilon$ and $\Upsilon^{\prime}$ are identical over the neighborhood.

Thus we have proved (3) and (4) of Theorem 4.I $I^{v+1}$ for the neighborhood of an arbitrary point of the cell $a$. That they hold for the entire cell $a$ may now be shown easily by use of the Heine-Borel theorem and the fact that any

† Osgood II, Chapter 1, §6, Satz 1. 
closed curve on $a$ can be deformed into coincidence with any given point of the curve. Hence the proof of Theorem 4.I ${ }^{\nu+1}$ is complete. We may therefore consider Theorem 4.I ${ }^{\nu}$ established for $\nu=1,2, \cdots, n-1$.

THEOREM 4.II. Let the functions $\Theta_{i}\left(x_{1}, \cdots, x_{n}\right), i=1, \cdots, m$, be real and analytic at the point $(x)=(0)$, vanishing there, but not vanishing identically. Then, after a suitable real change of coördinate axes which keeps the origin fixed, it is possible to enclose the origin in a closed region $\mathfrak{A}^{n}:\left|x_{i}\right| \leqq \mathfrak{a}_{i}, \mathfrak{a}_{i}>0$, which coincides with a complex $A^{n}$ of analytic cells, such that each of the loci $\Theta_{i}(x)=0$, in $\mathfrak{A}^{n}$, coincides with a sub-complex of $A^{n}$.

This follows from the proof of the preceding lemma, as the induction extends from $\nu=n-1$ to $\nu=n$, with certain simplifications. From the construction it follows that each of the loci $\Theta_{i}(x)=0$ is a sub-complex (compare construction of cells of the first class).

In connection with this theorem, see Lemma 3.I.

5. A property of analytic loci. We prove the following theorem:

THEOREM 5.I. Let $R$ be a connected n-dimensional open region of the real $\left(x_{1}, \cdots, x_{n}\right)$-space, and $M$ a closed sub-set of $R$. Let the functions $\Theta_{i}\left(x_{1}, \cdots, x_{n}\right) \not \equiv 0, i=1,2, \cdots, m$, be single-valued, real and analytic in $R$. Then a direction can be found as close to any given direction as we like (as measured by direction cosines), such that no locus $\Theta_{i}=0$ contains a straight line segment in $R$ through a point of $M$, in that direction.

By an internal element of $M$ we understand a set of real quantities $\left(x_{1}, \cdots, x_{n}, \xi_{1}, \cdots, \xi_{n}\right)$ such that $(x)$ is a point of $M ;\left(\xi_{1}, \cdots, \xi_{n}\right)$ are direction components of a line segment through $(x)$ in $R$, whose points lie on one of the loci $\Theta_{i}=0$; and

$$
\frac{1}{2} \leqq \xi_{1}^{2}+\cdots+\xi_{n}^{2} \leqq 1 .
$$

Thus, for some $k$, we shall have the identity in $t$

$$
\Theta_{k}\left(x_{1}+\xi_{1} t, \cdots, x_{n}+\xi_{n} t\right) \equiv 0,
$$

and conversely, any such identity for which $(x)$ is on $M$ and (5.1) is satisfied, defines an internal element.

Since we can expand the left hand side of (5.2) as a power series in $t$, the set of internal elements is found by equating the coefficients, say $C_{i}$, to zero. We denote by $T$ the locus thus obtained in $(x, \xi)$-space. We shall consider the part, say $S$, of $(x, \xi)$-space for which $(x)$ is on $M$, and ( $\xi$ ) satisfies (5.1). Here, and in a neighborhood of any such point, not all the coefficients $C_{i}$ can be identically zero. For in that case $T$ would contain line segments in all direc- 
tions through a point of $R$, so that $\Theta_{k}$ would be identically zero, contrary to hypothesis.

In a neighborhood of an arbitrary point of $S$, the locus $T$ is found by equating a finite number of the coefficients $C_{i}$ to zero. For, with any finite set we obtain a locus consisting of a finite number of configurations of various grades, and with each additional coefficient which is not identically zero on these configurations, some of them are replaced by configurations of lower grades.* As a finite number of such steps must reduce the locus to a single point or no point at all, unless, after a certain value of $i$, all the $C_{i}$ vanish identically on the configuration already obtained, our statement is correct.

Since we have only a finite number of analytic functions to consider in the neighborhood of any point of $S$, from Theorem 4 .II it follows that in any such neighborhood the locus $T$ is a complex $K_{i}$ of analytic cells. Since $S$ is closed, it is covered by a finite number of these complexes, overlapping (Heine-Borel theorem). Some of them will contain points not in $S$, in general.

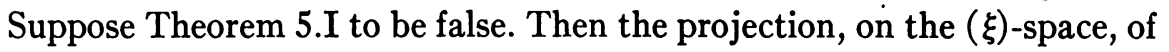
all the complexes $K_{i}$ must cover a neighborhood of a point in $(\xi)$-space representing the given direction. Consider the projections on $(\xi)$-space of all the closed cells of the $K_{i}$, starting with those of smallest dimensionality (zero), added together one by one. The first cell, say $B$, for which the sum covers a region in $(\xi)$-space must itself cover a region, since the preceding sum is a closed set.

Then the boundary of $B$ projects onto a point set containing no inner points. Hence the projection of $B$, less a neighborhood of the boundary, contains an open set. Next, we can divide the part of $B$ in question into any given number of closed parts (having certain common boundary points), at least one of which, by an argument given above, projects onto a set containing an inner point. Continuing this process of subdivision, we obtain a limit point, say $Q$, on $B$, such that the projection on $(\xi)$-space of any neighborhood of $Q$ on $B$ contains some inner points.

Since $B$ is analytic, in a sufficiently small neighborhood of $Q$ it is represented by equations of the form

$$
\begin{gathered}
x_{r+1}=x_{r+1}\left(x_{1}, \cdots, x_{r}, \xi_{1}, \cdots, \xi_{s}\right), \\
\cdot \cdot x_{n}=x_{n}\left(x_{1}, \cdots, x_{r}, \xi_{1}, \cdots, \xi_{s}\right), \\
\xi_{s+1}=\xi_{s+1}\left(x_{1}, \cdots, x_{r}, \xi_{1}, \cdots, \xi_{s}\right), \\
\left.\cdot \cdot x_{r}, \xi_{1}, \cdots, \xi_{s}\right), \\
\xi_{n}=\xi_{n}\left(x_{1}, \cdots, x_{r},\right.
\end{gathered}
$$

* Osgood II, Chapter 2, §17. 
where the functions on the right are analytic. Now if $s<n$, not all the partial derivatives of $\xi_{s+1}, \cdots, \xi_{n}$ with respect to $x_{1}, \cdots, x_{r}$ can be identically zero, as in that case $n-s$ of the $\xi$ 's would be dependent on $s$ of them and on nothing else, so that a neighborhood could not be covered in $(\xi)$-space. Suppose, then, that $\partial \xi_{s+1} / \partial x_{r}$ is not identically zero, and consider the locus determined by its vanishing, in the above neighborhood of $Q$. If the projection of that locus covers a region in $(\xi)$-space, then we fix attention on that locus, which is composed of cells of lower dimensionality than that of $B$, near $Q$. If, on the other hand, this is not true of its projection, it will be of that of the locus determined by the condition $\partial \xi_{s+1} / \partial x_{r} \neq 0$. Moreover, as before, we can even avoid a neighborhood of the locus for which $\partial \xi_{s+1} / \partial x_{r}=0$, and proceed as above to find another limit point, say $Q^{\prime}$, on $B$, having the same properties as $Q$, but with $\partial \xi_{s+1} / \partial x_{r} \neq 0$ for $Q^{\prime}$. By the implicit function theorem, in a sufficiently small neighborhood of $Q^{\prime}$ the points satisfying $\xi_{s+1}=\xi_{s+1}\left(x_{1}, \cdots, \xi_{s}\right)$ are given by taking $x_{r}$ as an analytic function of $\left(x_{1}, \cdots, x_{r-1}, \xi_{1}, \cdots, \xi_{\text {s }}\right.$, $\left.\xi_{s+1}\right)$ and substituting in the other equations of (5.3). We then have the locus (5.3), near $Q^{\prime}$, expressed in a new way, with one more of the $\xi$ 's now appearing as an independent variable.

If we continue to apply the above method, at each step we will therefore either obtain a cell of lower dimensionality, or introduce another $\xi_{i}$ as independent variable. Since a single point cannot project onto a set containing an inner point, eventually the former must cease to occur, so that all the $\xi$ 's will finally appear as independent variables. If we then set all the independent variables except the $\xi$ 's equal to constants, we shall have an analytic cell of dimension $n$,

$$
x_{i}=x_{i}\left(\xi_{1}, \cdots, \xi_{n}\right) \quad(i=1,2, \cdots, n),
$$

part of $T$. Then by (5.2) we have

$$
\Theta_{k}\left[x_{1}\left(\xi_{1}, \cdots, \xi_{n}\right)+\xi_{1} t, \cdots, x_{n}\left(\xi_{1}, \cdots, \xi_{n}\right)+\xi_{n} t\right] \equiv 0
$$

for every $\xi_{1}, \cdots, \xi_{n}, t$ in a certain $(n+1)$-dimensional region. Since $\Theta_{k}$ is not identically zero in its arguments, for any value of $t$ in a certain interval the Jacobian

$$
\frac{\partial\left(x_{1}+\xi_{1} t, \cdots, x_{n}+\xi_{n} t\right)}{\partial\left(\xi_{1}, \cdots, \xi_{n}\right)} \equiv 0 .
$$

But, since the highest power of $t$ in the expansion of the determinant has coefficient unity, this is impossible. Hence Theorem 5.I is true.

COROLlARY 5.II. In Theorem 5.I, the loci $\Theta_{k}=0$ may be replaced by a finite number of complexes of analytic cells such as arise in Theorem 4.II, which may overlap; and the conclusion will be valid. 
This follows immediately from the kind of proof used for Theorem 5.I.

6. Analytic loci in the large. We prove the following theorem:

THEOREM 6.I. Let $R$ be a connected open region of a real $n$-dimensional number-space $S^{n}$, and $M$ a closed sub-set of $R$; and let $\Theta_{1}, \cdots, \Theta_{m}$ be real single-valued analytic functions in $R$, not identically zero. Then $M$ can be embedded in a complex $K \subset R$ of analytic cells, such that each locus $\Theta_{j}=0$ on $K$ coincides with a sub-complex of $K$.

Let $S^{n}$ be covered by a lattice of $n$-cubes, denote by $K^{n}$ the set of closed cubes of the lattice which have at least one point in common with $M$; and suppose that the lattice is so fine that $K^{n} \subset R$. Let the equations of the $(n-1)$ faces of the cubes of $K^{n}$ be adjoined to the given equations, and continue to denote the amplified set by $\Theta_{1}=0, \cdots, \Theta_{m}=0,-$ an inconsequential change of notation. Let $T^{n-1}$ be the locus of real points of $K^{n}$ at which at least one $\Theta_{j}$ vanishes.

In our proof we shall introduce what will be called a proper system of axes in $S^{n}$, namely, one for which the statements $\left(6.1^{\nu}\right),\left(6.2^{\nu}\right),\left(6.3^{\nu}\right)$ below are valid, $\nu=n-1, n-2, \cdots, 1,0$. In these statements, $K^{\nu}$ is the projection of $K^{n}$ upon the $\left(x_{1}, \cdots, x_{\nu}\right)$-space, $K^{0}$ the origin.

$\left(6.1^{n-1}\right) A$ line through any point $P_{n-1}$ of $K^{n-1}$ parallel to the $x_{n}$-axis cuts $T^{n-1}$ in a finite set $\left\{P_{n}\right\}$ of real points $P_{n}$.

When $P_{n-1}^{1}$ is given on $K^{n-1}$, there are two possibilities: either the set of values of $x_{n}$ determined by the set $\left\{P_{n}\right\}$ corresponding in the above fashion with $P_{n-1}$ will form, for all $P_{n-1}$ sufficiently near to $P_{n-1}^{1}$, a set of distinctvalued analytic functions of $\left(x_{1}, \cdots, x_{n-1}\right)$; or else this is untrue however small a neighborhood of $P_{n-1}^{1}$ be taken. In the second case, we assign $P_{n-1}^{1-1}$ to a new locus $T^{n-2} \subset K^{n-1}$. (Evidently $T^{n-2}$ contains the projections on $K^{n-1}$ of all the $(n-2)$-dimensional faces of the $n$-cubes of $K^{n}$.)

(6.1 $\left.{ }^{\nu}\right) A$ line through any point $P_{\nu}$ of $K^{\nu}$ parallel to the $x_{\nu+1}$-axis cuts $T^{\nu}$ in a finite number of points.

Let $\left\{P_{\nu+1}\right\}$ be this finite set of intersections determined by $P_{\nu}$ on $T^{\nu}$. Through each $P_{\nu+1}$ pass a parallel to the $x_{\nu+2}$-axis: it will cut $T^{\nu+1}$ in a finite set of points $P_{\nu+2}$, by $\left(6.1^{\nu+1}\right)$. Through each $P_{\nu+2}$ pass a parallel to the $x_{\nu+3^{-}}$ axis: they will all cut $T^{\nu+2}$ in a finite set of points $P^{\nu+2}$, by $\left(6.1^{\nu+2}\right) . \cdots$ Continuing in this manner, we finally have a finite set $\left\{P_{n}\right\}$ of points on $T^{n-1}$ determined by the given point $P_{\nu}$ of $K^{\nu}$.

When $P_{\nu}^{1}$ is given on $K^{\nu}$, there are two alternatives: either the set of values of $x_{\nu+\mu}$ determined by the points $\left\{P_{\nu+\mu}\right\}$ obtained in the above fash- 
ion from $P_{\nu}$ form, for all $P_{\nu}$ sufficiently near to $P_{\nu}{ }_{\nu}$, a set of distinct-valued analytic functions of $\left(x_{1}, \cdots, x_{\nu}\right)$, and this for each $\mu=1,2, \cdots, n-\nu$; or else this is untrue for at least one of these values of $\mu$. In the second case we assign $P_{\nu}^{1}$ to a new locus $T^{\nu-1} \subset K^{\nu}$. Evidently $T^{\nu-1}$ contains the projections on $K^{\nu}$ of all the $(\nu-1)$-dimensional faces of the $n$-cubes of $K$.

$\left(6.2^{\nu}\right)$ Any point $P_{\nu}^{1}$ on $K^{\nu}$ being given, it will be possible, maintaining the original $x_{n}, x_{n-1}, \cdots, x_{\nu+1}$-axes of the proper system, but possibly introducing new $x_{\nu}, \cdots, x_{1}$-axes (dependent on $P_{\nu}^{1}$ ), to construct rectangles $H^{n}$ centering at the $P_{n}$ 's of the finite set $\left\{P_{n}\right\}$ determined as above by $P_{\nu}{ }_{\nu}$, all with edges parallel to the axes, and corresponding dimensions equal, and such that conditions (a) and (b) below are satisfied by the r-rectangles $H^{r}$ in which the $H^{n}$ 's project on $S^{r}(r=n-1, n-2, \cdots, \nu)$ :

(a) By a method essentially that of $\$ 4$, each $H^{r}$ may be covered by complexes of analytic cells, the algebroid polynomials $F_{i}{ }^{(t)}\left(x_{1}, \cdots, x_{t}\right)$ used here being obtained as in \$4, in the process of forming successive resultants and discriminants, starting with our given $\Theta_{i}$ 's in the different $H^{n}$ 's. But (cf. below) here the $F$ 's need not be singular at the centers of the rectangles. This construction can be so performed that, if $L^{r}$ is the locus in all the $H^{r+1}$ 's at which the functions $F^{(r+1)}$ vanish, $L^{r}$ will coincide with a sub-complex of the complex covering the $H^{r+1}$ 's in question.

(b) The part of $T^{r}$ in these $H^{r+1}$ 's shall coincide with a sub-complex of the complex covering $L^{r}$.

(6.3 $)$ The part of $T^{\nu-1}$ in $H^{\nu}$ is a sub-set of $L^{\nu-1}$.

We now prove the existence of a proper system of axes, using induction. Suppose directions have been determined for the $x_{n}, x_{n-1}, \cdots, x_{\nu+1}$-axes for which $\left(6.1^{n-1}\right),\left(6.2^{n-1}\right),\left(6.3^{n-1}\right), \cdots,\left(6.1^{\nu}\right),\left(6.2^{\nu}\right),\left(6.3^{\nu}\right)$ are satisfied. We proceed to determine a direction for the $x_{\nu}$-axis which will further render valid $\left(6.1^{\nu-1}\right),\left(6.2^{\nu+}\right)$ and $\left(6.3^{\nu-1}\right)$.

For any point $P_{\nu}$ of $K^{\nu}$, we have a corresponding $H^{\nu}$, according to $\left(6.2^{\nu}\right)$, and a finite set of $H^{\nu+1}$ 's which project upon it, on each of which is determined a finite set of algebroid polynomials $F_{i}^{(\nu+1)}$. We form the set $R_{i j}^{(\nu)}\left(x_{1}, \cdots, x_{v}\right)$ of the resultants of all these $F_{i}^{(\nu+1)}$ 's, and the discriminants of them taken in pairs, where both members of a pair are defined over the same $H^{\nu+1}$. Next we treat these $R_{i j}^{(v)}$ as in a similar case in $\$ 4$ (cf. below), obtaining equations of the form (4.6), (4.7), but with $x_{\nu}$ replaced by $\left(x_{\nu}-x_{\nu}{ }^{0}\right)$, where $x_{\nu}{ }^{0}$ is the value of $x_{\nu}$ at $P_{\nu}$, and with the vertex at the projection of $P_{\nu}$ on $K^{\nu-1}$. This procedure may involve decreasing the size of $H^{\nu}$, and a change of $x_{1}, \cdots, x_{\nu}-$ axes. Thus we have algebroid polynomials determined in $\left(x_{\nu}-x_{\nu}{ }^{0}\right)$; which we 
proceed to rewrite as algebroid polynomials in $x_{v}$, with the result that the new algebroid polynomials are no longer necessarily singular. The latter are taken as the functions $F_{i}^{(v)}$ of (6.2). For the $H^{v}$ in question, the locus $\overline{L^{v-1}}$ is then determined by the vanishing of these functions $F_{i}(v)$.

By the Heine-Borel theorem, a finite set of such $H^{\nu}$ 's can be obtained which will cover all of $K^{\nu}$. Let $\left\{H^{\nu}\right\}_{1}$ denote such a set. According to Corollary 5.II, a direction exists such that no line segment in that direction cuts the totality of the corresponding $L^{\nu-1}$ 's in a line segment and hence, since the $L^{v-1}$ 's are defined by analytic equations, in more than a finite set of points. We choose such a direction for the $x_{\nu}$-axis, and proceed to show that it satisfies $\left(6.1^{\nu-1}\right),\left(6.2^{\nu-1}\right)$ and $\left(6.3^{\nu-1}\right)$.

Consider any line in $K^{\nu}$ parallel to the $x_{\nu}$-axis. Any intersection of that line with $T^{\nu-1}$ must be interior to one of the set of overlapping $H^{\nu}$ 's mentioned in the preceding paragraph. According to the last paragraph, the line will cut all the $L^{\nu-1}$ 's in a finite set of points. Since, by $\left(6.3^{\nu}\right)$, the part of $T^{\nu-1}$ in any $H^{v}$ is a sub-set of the corresponding $L^{\nu-1}$, it therefore follows that the line cuts $T^{\nu-1}$ in only a finite number of points. In other words, $\left(6.1^{\nu-1}\right)$ is proved.

Now let $P_{\nu-1}$ be any point of $K^{\nu-1}$, and let us establish $\left(6.2^{\nu-1}\right)$. Choosing any directions for the $x_{1}, \cdots, x_{\nu-1}$-axes, consider all the points $P_{r}, r=\nu$, $\nu+1, \cdots, n$, determined as described above following $\left(6.1^{\nu}\right)$, with $\nu$ replaced by $\nu-1$. If the corresponding rectangles $H^{r}$ are taken small enough, they will all lie in the $H^{r}$ 's determined by the rectangles $\left\{H^{\nu}\right\}_{1}$; in fact, if one of the present $H^{\nu}$ 's is taken small enough to be interior to one of the $H^{\nu}$ 's of $\left\{H^{\nu}\right\}_{1}$, its corresponding $H^{r}$ 's can be made to be interior to the $H^{r}$ 's determined by the $H^{\nu}$ of $\left\{H^{\nu}\right\}_{1}$ in question. It is then easily seen that the present $L^{r-1}$ 's will be sub-sets of the $L^{r-1}$ 's of the latter $H^{r}$ 's, so that the assigned $x_{n}, x_{n-1}, \cdots, x_{\nu}$-directions, for which these $L^{r-1}$ 's are cut in only finite numbers of points, have the same property for the present $H^{r}$ 's.

Let us now compare the present situation with that in $\$ 4$. Corresponding to $H^{\nu-1}$ is $\mathfrak{A}^{\nu-1}$ of $\$ 4$. (See Theorem 4.I.) Corresponding to $\mathfrak{A}^{\nu}$ of $\$ 4$ we now have a finite set of $H^{\nu}$ 's, which project upon $H^{\nu-1}$. For each of these $H^{\nu}$ 's we have a finite set of $H^{\nu+1}$ 's, as compared with the single $\mathfrak{A}^{\nu+1}$ of $\$ 4$; and so on. Another difference is that the functions $F_{i}{ }^{(r)}$ of this section are algebroid polynomials in general non-singular, while those in $\$ 4$ are singular. However, the latter difference is of no consequence, as the work of $\$ 4$ does not depend upon the singularity of the algebroid polynomials (cf. Corollary 2.IX and Lemma 2.X), this being a mere convenience. Consideration of $\$ 4$ now shows that if the $x_{1}, \cdots, x_{\nu-1}$-directions are chosen properly, its procedure can be extended to the present case, with the following modification. When $H^{\nu-1}$ is 
covered by a complex of analytic cells, we then proceed, as in the construction of Theorem 4.I $I^{\nu-1}$, to cover by a complex of analytic cells each of the $H^{\nu}$ 's which projects upon $H^{\nu-1}$, making use of the complex covering $H^{\nu-1}$. Then, using the complex covering each of these $H^{\nu}$ 's, we cover by complexes of analytic cells all the $H^{\nu+1}$ 's which project upon it. Proceeding in this way, we finally have all the $H^{r}$ 's, $r=\nu-1, \nu, \cdots, n$, covered by complexes of analytic cells. Observing the definition of $L^{r}$ as the locus where the functions $F_{i}{ }^{(r+1)}$ vanish, and comparing with $\S 4$, we see that the $L^{r}$ in any $H^{r+1}$ is a sub-complex of the complex of analytic cells just constructed, so that $\left(6.2^{\nu-1}(\mathrm{a})\right)$ is established. Next we prove $\left(6.2^{\nu-1}(\mathrm{~b})\right)$.

We take all the dimensions of the $H^{n}$ 's corresponding to the given point $P_{\nu-1}^{1}$ so small that the locus $T^{\nu-2}$ in $H^{\nu-1}$ is determined solely by the part of $T^{n-1}$ in the $H^{n}$ 's. Let $W$ be a cell of $L^{\nu-1}$ in some $H^{\nu}$ corresponding to $P_{\nu-1}^{1}$; and suppose that some point $Z$ of $W$ is not on $T^{\nu-1}$. We shall show that no point of $W$ can then be on $T^{\nu-1}$. Since $L^{\nu-1}$ is composed of cells of the first class (see definition of $L^{r}$, and the construction of $\$ 4$ ); it follows from $\left(6.3^{\nu}\right)$ that no cell of the second or third class can have a point on $T^{\nu-1}$; hence we may assume that $W$ is of the first class. First, suppose $W$ is a $(\nu-1)$-cell. Then, by methods similar to those used to prove Lemma 2.X, and with the use of the hypothesis about $Z$, it can be shown without difficulty that the set of cells of $L^{\nu}$ on $T^{\nu}$ (in the $H^{\nu+1}$ 's projecting on $H^{\nu}$ ) determined by $W$ and its two incident $\nu$-cells in $H^{\nu}$, determine a set of functions real, distinct-valued and analytic over those three cells; so that no point of $W$ can lie on $T^{\nu-1}$ (cf. the definition of $T^{\nu-1}$ ). The proof involves a region in the space of the complex variables $x_{1}, \cdots, x_{\nu}$, neighboring the locus of $W$, and consists in proving that a certain locus which contains $W$ is a locus of removable singularity.* The result for the case that $W$ is of dimension less than $\nu-1$, is an easy consequence of the result for $(\nu-1)$-cells, and the same theorems on removable singularities. Now $T^{\nu-1}$ is a closed set, as follows from its definition. From $\left(6.3^{\nu}\right)$ and the result just proved it therefore follows that the part of $T^{\nu-1}$ in $H^{\nu}$ is covered by (coincides with) a sub-complex of the complex which covers the corresponding $L^{\nu-1}$. Consequently $\left(6.2^{\nu-1}(\mathrm{~b})\right)$ is established for $r=\nu-1$. As similar treatment applies for larger values of $r,\left(6.2^{\nu-1}\right)$ is proved.

From the results just established, we infer that if, near a given point $P$ of $L^{\nu-1}, L^{\nu-1}$ is given by equating $x_{\nu}$ to a single real analytic function of $\left(x_{1}, \cdots, x_{\nu-1}\right)$, then in a sufficiently small neighborhood of $P$ either no point of $L^{\nu-1}$, or else every point of $L^{\nu-1}$, is a point of $T^{\nu-1}$. Since, by $\left(6.3^{\nu}\right), T^{\nu-1}$ is a sub-set of $L^{\nu-1}$, and similar statements can be made of $T^{\nu}, T^{\nu+1}, \cdots, T^{n-1}$,

*Cf. Osgood II, Chapter 3, $\S 5$, for theorems on removable singularities. 
$L^{\nu}, L^{\nu+1}, \cdots, L^{n-1}$, it therefore follows that our $T^{\nu-2}$ (locus of singularities of $T^{\nu-1}, T^{\nu}, \cdots, T^{n-1}$ ) must be a sub-set of $L^{\nu-2}$ (which contains the locus of singularities of $\left.L^{\nu-1}, L^{\nu}, \cdots, L^{n-1}\right)$. Thus $\left(6.3^{\nu-1}\right)$ is established.

As $\left(6.1^{n-1}\right),\left(6.2^{n-1}\right),\left(6.3^{n-1}\right)$ admit simplified versions of the preceding proofs, we can now consider $\left(6.1^{\nu}\right),\left(6.2^{\nu}\right),\left(6.3^{\nu}\right)$ to be established inductively for $\nu=n-1, n-2, \cdots, 1$, with a proper system of axes.

Now we say that $T^{0}$ contains only a finite number of points. For, about any point $P$ of $K^{1}$ we can take a closed segment $H^{1}$ to which $\left(6.1^{1}\right),\left(6.2^{1}\right)$, (6.3) apply. Hence, in $H^{1}, T^{0}$ is a sub-complex of the corresponding $L^{0}$; and as the latter contains only isolated points, the same is true of $T^{0}$. As $T^{0}$ is a closed set on $K^{0}$, it follows that $T^{0}$ contains only a finite number of points.

We can now complete the proof of Theorem 6.I. First we cover $K^{1}$ by the complex whose 0 -cells are the points of $T^{0}$. Next we cover $K^{2}$ by a complex, as follows: Over each $\nu$-cell $(\nu=0,1)$, the corresponding points on $T^{1}$ form a finite number of $\nu$-cells, analytic if $\nu=1$, which we designate as of the first class. The points of $K^{2}$ which project on a $\nu$-cell of $K^{1}$, and are between two successive (in the order of values of $x_{2}$ ) $\nu$-cells of the first class, form a $(\nu+1)$-cell, called a cell of the third class. The totality of 0 -, 1 - and 2-cells thus determined on $K^{2}$ form a complex covering $K^{2}$, as any details of the proof that these cells form a complex are the same as those in $\$ 4$.

Next we cover $K^{3}$ by a complex of cells of the first and third class Over each $\nu$-cell $(\nu=0,1,2)$ of $K^{2}$ are determined a finite number of $\nu$-cells of the first class and of $(\nu+1)$-cells of the third class, where the $\nu$-cells of the first class all lie on $T^{2}$, and are analytic if $\nu>0$, as follows from the definitions of the sets $T^{r}$. This process is continued till finally we obtain an $n$-dimensional complex of cells, some of which, in general, will not be in $K$. Upon dropping the latter cells, we have the required complex covering $K$. The sub-complex determined by an equation $\Theta_{i}=0$ will consist of cells of the first class, hence is of dimension not exceeding $n-1$. The proof of Theorem 6.I is now complete.

THEOREM 6.II. Let $\Theta_{i}\left(z_{1}, \cdots, z_{n}\right), i=1, \cdots, m$, be functions of the complex variables $z_{1}, \cdots, z_{n}$, single-valued and analytic over an open region $R$ of the $2 n$-space of the complex variables, and not identically zero. Then if $M$ is any closed sub-set of $R, M$ can be embedded in the interior of a sub-set $K$ of $R$, where $K$ is a 2n-dimensional complex of analytic cells, such that the locus, in $K$, of each of the equations $\Theta_{i}=0$ is a sub-complex of K. Furthermore, the sub-complex defined by the simultaneous solutions, or the totality of solutions, of any sub-set, or of all, of the equations, is of even dimensionality.

Writing $z_{k}=x_{k}+(-1)^{1 / 2} y_{k}, k=1,2, \cdots, n$, we will have 
$\Theta_{j}\left(z_{1}, \cdots, z_{n}\right)=\Phi_{j}\left(x_{1}, \cdots, x_{n} ; y_{1}, \cdots, y_{n}\right)+(-1)^{1 / 2} \Psi_{j}\left(x_{1}, \cdots, y_{n}\right)$,

where $\Phi_{j}$ and $\Psi_{j}$ are real analytic functions of $\left(x_{1}, \cdots, x_{n} ; y_{1}, \cdots, y_{n}\right)$ over the region of real $\left(x_{1}, \cdots, y_{n}\right)$-space corresponding to $R$. This follows readily from the absolute convergence properties of power series. The locus in question is now the real locus determined by replacing the equation $\Theta_{j}=0$ by the simultaneous equations $\Phi_{j}=0$ and $\Psi_{j}=0$. Theorem 6.II then follows from Theorem 6.I, except for the last part of the conclusion, which we now prove.

Let $L$ denote the sub-complex mentioned in the last sentence of the theorem, and let $a^{t}$ be one of its cells of highest dimensionality, namely $t$. If $t=0$ there is nothing to prove, therefore we suppose that $t>0$. Now $L$ consists of a finite number of configurations of various grades, ${ }^{*}$ near any point on it. Let $P$ be any point on $a^{t}$; nearby on one of the configurations, say $G$, of the highest grade for the neighborhood, can be found a point $Q$ near which $L$ is given by taking certain of the variables $z_{k}$ as analytic functions of the others as independent variables. It follows that a small neighborhood of $Q$, on $L$, is covered by a cell of even dimensionality (twice the grade of $G$ ). Since $a^{t}$ is a cell of the highest dimension for $L, Q$ must be on $a^{t}$. Thus we have a neighborhood of $Q$ on $a^{t}$ covered in one-to-one and continuous manner by a cell of even dimension. By the theorem of invariance of dimensionality $\dagger$ it therefore follows that $a^{t}$ is of the same even dimension. Consequently $L$ is of even dimension, and the theorem is proved.

7. A topological property of analytic loci. We prove the following theorem:

THEOREM 7.I. Under the notation of Theorem (6.I), let $L$ denote the boundary $(\bmod 2)$ of $K$. (Thus no point of $M$ is on $L$.) Let $H$ denote the sub-complex of $K$ determined by the simultaneous solutions, or totality of solutions, of any sub-set, or of all, of the equations $\Theta_{i}=0$. Let $H^{\prime}$ denote the complex consisting of all the (n-1)-cells of $H$ and the cells on the boundaries of the latter. Then if $H^{\prime}$ is not vacuious, it is a cycle $(\bmod 2 ; L)$, and determines an oriented $(n-1)$ cycle $(\bmod L)$ in which each of its $(n-1)$-cells appears (oriented) with coefficient plus or minus one.

First we note that we may assume that $H$ is the locus of the totality of solutions of the equations $\Theta_{i}=0$. For the simultaneous real solutions of a set of real equations are the same as the real solutions of the set of equations obtained by equating to zero the sum of the squares of the left hand members

* Osgood II, Chapter 2, $\$ 17$, second Weierstrass theorem.

† L. E. J. Brouwer, Beweis der Invarianz der Dimensionszahl, Mathematische Annalen, vol. 70 (1911), pp. 161-165. Cf. Lefschetz, loc. cit., p. 99. 
of the equations in question; and the property of being a cycle $(\bmod 2)$ is independent of the particular complex used to cover the locus. ${ }^{*}$ The final conclusion of the theorem will be a consequence of the fact that $H^{\prime}$ is a cycle $(\bmod 2 ; L)$.

Now the locus $H^{\prime}=H^{\prime n-1}$ in question consists of cells of the first class, as follows from the construction of the preceding section. Let $W^{n-2}$ be any $(n-2)$-cell of $H^{\prime n-1}$, and $W_{1}^{n-2}$ the projection of $W^{n-2}$ on $S^{n-1}$, the plane of the variables $\left(x_{1}, \cdots, x_{n-1}\right)$. Let $\Sigma^{2}$ be the 2-plane determined by the normal to $W_{1}^{n-2}$ in $S^{n-1}$ at some point $P_{1}$, and the line through $P_{1}$ parallel to the $x_{n}$-axis. Let $P$ be the point of $W^{n-2}$ which projects onto $P_{1}$. Then near $P$ in $\Sigma^{2}$ the locus $J^{1}$ where the functions $\Theta_{i}$ vanish consists of the points of $H^{\prime n-1}$ near $P$ in $\Sigma^{2}$, with $P$ as the only point on $W^{n-2}$ in $\Sigma^{2}$.

Each $(n-1)$-cell of $H^{\prime n-1}$ incident with $W^{n-2}$ determines a 1 -cell on $J^{1}$ in $\Sigma^{2}$, near $P$, incident with $P$. This follows from consideration of the determination of the $(n-1)$-cells of $H^{\prime n-1}$ by setting $x_{n}$ equal to distinct analytic functions of $\left(x_{1}, \cdots, x_{n-1}\right)$. Now, the locus in a real 2 -space where a set of analytic functions vanish has the property that at each point on it there are an even number of analytic 1-cells abutting. This can be proved by use of the Weierstrass preparation theorem; and the parametric representation for the locus defined by equating to zero an analytic function of two variables. $\dagger$ Since, then, we have an even number of 1 -cells abutting on $P$ in $\Sigma^{2}$, it follows that $H^{\prime n-1}$ must have an even number of $(n-1)$-cells abutting on $W$. This completes the proof that $H^{\prime n-1}$ is a cycle $(\bmod 2 ; L)$.

The final result of the theorem now follows easily from the following considerations of analysis situs: euclidean $n$-space contains no non-bounding cycles $(\bmod 2)$; it is orientable, where the point-set boundary of any sum of oriented $n$-cells, each taken once, involves the same $(n-1)$-cells as appear in the boundary $(\bmod 2)$ of the sum.

* A. B. Brown, Topological invariance of sub-complexes of singularities, American Journal of Mathematics, vol. 54 (1932), pp. 117-122; Corollary 4.

† Osgood, Lehrbuch der Funktionentheorie, vol. I, Chapter 8, §§12-14.

Columbia University, NEW YORK, N. Y. 